\title{
CYTOPLASMIC INHERITANCE OF THE SELFING FACTOR IN THE MYXOMYCETE DIDYMIUM IRIDIS
}

\author{
JOHN J. YEMMA \\ Department of Biology, Youngstown State University, Youngstown, Ohio, U.S.A. \\ and \\ C. D. THERRIEN and SHARON VENTURA \\ Department of Biology, The Pennsylvania State University, University Park, Pa, U.S.A.
}

Received 16.iv.73

\section{SUMMARY}

The mechanism by which the selfing factor is inherited in the heterothallic myxomycete Didymium iridis has been investigated. A non-selfing isolate, Honduran $A^{2}$ was crossed with two selfing isolates, Panamanian 2-4 and Panamanian 2-7. The latter two isolates bear the mating alleles $A^{7}$ and $A^{8}$ respectively. Meiotic segregants were then analysed for selfing frequency. Mating types were used as a nuclear marker. The capacity to self was inherited by all segregants, with the $A^{2}$ segregant selfing at a higher frequency than either the $A^{7}$ or $A^{8}$ segregant. The incidence of selfing increases in the $A^{2}$ segregant as it is successively recloned. In the $\mathrm{F}_{3}$ generation the frequency of selfing is observed to be 100 per cent. A cytoplasmic factor is postulated with the Honduran $A^{2}$ mating type being more susceptible to its action than either of the other two mating types.

\section{Introduction}

CompatibiLITy systems in the plasmodial slime molds, the myxomycetes, have been the subject of intensive genetic analyses during the past 10 years. Most notable have been those investigations with Physarum polycephalum (Dee, 1962, 1966), Didymium iridis (Collins, 1961, 1963, 1965; Collins and Ling, 1964, 1968; Mukherjee and Zabka, 1964), Physarum pusillum (Collins, 1962), Physarum flavicomum (Henney, 1967; Henney and Henney, 1968) and Physarum rigidum (Henney and Henney, 1968).

In general, two principal types of compatibility systems appear to be functional in the myxomycetes. Homothallism, in which case uninucleate cells, either myxamoebae or flagellated swarmers, derived from a single spore isolate are capable of fusing with the resultant formation of a multinucleate coenocyte, the plasmodium, without the presence of cells of a compatible mating type; and heterothallism, in which case single spore derived clones are normally self-sterile, but cross compatible with clones derived from spores of other mating types. Additionally, at least two species, Physarum polycephalum (Dee, 1966) and Didymium iridis (Collins, 1963; Collins and Ling, 1964; Mukherjee and Zabka, 1964), appear to be characterised by a single locus, multiple allelic system of compatibility. With the latter species, $D$. iridis, at least eight different mating alleles have been identified. For normal plasmodial formation to occur in $D$. iridis it is only necessary that cells of any two mating types, i.e. $A^{1} \times A^{2}, A^{1} \times A^{7}, A^{2} \times A^{7}$, be crossed under appropriate culture conditions.

It should be noted here that in some cases single cell derived clones of 
myxamoebae can form plasmodia without the necessity of gametic fusion or nuclear phase alternance. Plasmodia thus formed are said to result from apogamy, and this has been reported to occur in several instances (Gehenio and Luyet, 1950; Kerr, 1967). The latter study, however, was with a mutant strain of the particular species being studied, Didymium nigripes, and it is generally acknowledged that the wild type strain of this species is homothallic (Kerr, 1961).

One other departure from the usual compatibility mechanism has been reported elsewhere (Collins and Ling, 1968; Yemma and Therrien, 1972) and is the subject of this investigation. That is the phenomenon by which plasmodia occasionally arise in clones of heterothallic species, or in common mating type crosses involving isolates of those species. This type of plasmodial formation has been termed "selfing" Collins and Ling, 1968). Subsequent investigations suggest that plasmodia produced in this manner are in fact diploid, as are those produced by crosses between compatible mating types, but that there are certain specific alterations of the nuclear cycle, especially with regard to the relative times of $G_{1}, S$ and $G_{2}$ periods of the mitotic cycle (Yemma and Therrien, 1972). Also of interest in the latter study was our observation that the selfing factor could be concentrated by simply recloning sporangia produced from selfed plasmodia. A possible explanation for this observed behaviour is that some cytoplasmic genetic factor, perhaps a cytoplasmic gene or a virus, promotes selfing. The factor may become concentrated because those myxamoebae which arose from spores that had been cleaved with the factor were preferentially functional in subsequent plasmodial formation. Alternatively, the compatibility locus may function in the same manner as an "operon ", as suggested by Collins and Ling (1968), and selfing may result from a mutation in either the operator or regulator gene. Such a mutation would permit constitutive synthesis of some factor necessary for compatibility, and at the same time result in no detectible change in the mating type (structural gene). Certainly this could account for the fact that selfing does not result in a mating type change within a single spore derived clone (Collins and Ling, 1968; Yemma and Therrien, 1972).

A heterokaryon test, in which one makes the appropriate crosses, then determines whether the factor is inherited as a Mendelian character (nuclear gene) or as a cytoplasmic factor should be useful in clarifying the perplexing problem. For instance, in a cross between a clone which is normally not capable of selfing, i.e. $A^{2}$, and a selfing clone, Pan 2-4 $\left(A^{7}\right)$, one should be able to analyse the meiotic segregants and determine if the selfing factor is inherited as a nuclear of cytoplasmic gene.

\section{Materials and methods}

All isolates of Didymium iridis (Ditmar) Fries used in this investigation were made available to us by $\mathrm{Dr} \mathrm{O}$. R. Collins, University of California, Berkeley. The isolates were designated $A^{2}$, Pan 2-4 (a selfing isolate bearing the $A^{7}$ mating allele) and Pan 2-7 (a selfing isolate bearing the $A^{8}$ mating allele). The $\mathrm{A}^{2}$ mating type is a non-selfing isolate, hence it was used as the tester clone in this investivation.

All isolates were cultured with the bacterium Escherichia coli on halfstrength Difco Corn Meal Agar (CM/2). The medium was prepared by 
dissolving 8.5 g Difco Corn Meal Agar and 8.0 g Difco Agar in distilled water, then bringing the final volume to 1 litre prior to being autoclaved. Crosses were made by incoculating myxamoebae of the appropriate mating types to GM/2 agar that had been pre-inoculated with $E$. coli. The bacteria were permitted to grow for a period of 24 hours at $37^{\circ} \mathrm{C}$. in order to form a "lawn" on the agar surface prior to the inoculation of myxamoebae. As plasmodia appeared they were transferred to fresh CM/2 plates and there allowed to sporulate. Sporulation was generally enhanced by providing an artificial day-night cycle in the incubator.

The following procedure was followed for the analysis of meiotic segregants. Whole sporangia were first removed from the CM/2 agar, then streaked in a drop of sterile distilled water on three per cent Difco Agar. After the water had been absorbed by the agar, single spores were isolated under a stereomicroscope at a magnification of $60 \times$. Dumont number 5 stainless-steel forceps were used to make the spore isolations. The isolated spores were then transferred to $60 \times 15 \mathrm{~mm}$ petri dishes containing $\mathrm{CM} / 2$, and a drop of the $E$. coli suspension added. Large clones of myxamoebae generally developed within a week. If no myxamoaebae were in evidence within the period of 1 week the spores were assumed to be inviable and the cultures set aside to be examined at a later date. Further examination of these cultures demonstrated that in no case did clones appear after the initial time period. Cultures of myxamoebae (derived from single spore isolates) were then recloned to 50 plates each and incubated at $23^{\circ} \mathrm{G}$. Again the plates of CM/ 2 had been pre-inoculated 24 hours previously with a suspension of $E$. coli. The cultures were then scored at the end of 7-, 14- and 28-day periods for the presence of plasmodia (selfing).

\section{Results}

All isolates were initially replica plated to 100 plates each of $\mathrm{CM} / 2$ which had been pre-incubated with $E$. coli. The percentages of clonally produced plasmodia (selfing) in the initial transfers are shown in table 1 .

TABLE 1

\begin{tabular}{ccc}
\multicolumn{3}{c}{ TABLE 1 } \\
\multicolumn{3}{c}{ Initial frequency of selfing } \\
$A^{2}$ & Pan 2-4 & Pan 2-7 \\
$n=100$ & $n=100$ & $n=100$ \\
$0 \cdot 0 \%$ & $20 \cdot 0 \%$ & $10 \cdot 0 \%$
\end{tabular}

The Honduras isolate (Collins, 1963), $A^{2}$, failed to self, whereas the Panamanian isolates, Pan 2-4 and Pan 2-7, showed varying degrees of selfing. The latter two isolates bear the mating alleles $A^{7}$ and $A^{8}$ respectively. The failure of the $A^{2}$ isolate to self was anticipated. In fact, no reports are to be found in the literature which might indicate that this isolate has the capacity to yield plasmodia from individual clones. This non-selfing $A^{2}$ isolate was essential for the heterokaryon analysis.

After the initial frequency of selfing was determined, both Panamanian isolates were crossed with myxamoebae bearing the $A^{2}$ mating allele. In each case 50 crosses were made. The presence of plasmodia in all crosses indicated that the isolates used in this investigation, Pan 2-4 $\left(A^{7}\right)$ and $\operatorname{Pan} 2-7$ $\left(A^{8}\right)$, both bear mating alleles that are compatible with the $A^{2}$ mating allele 
borne by the Honduran isolate. One would expect just these results in a one-locus, multiple allelic system of compatibility such as that associated with $D$. iridis (Collins, 1963).

Spores resulting from these crosses (Pan 2-4 $\left(A^{7}\right) \times A^{2}$ and Pan 2-7 $\left(A^{8}\right) \times\left(A^{2}\right)$ were then isolated. To determine the mating types of the viable

TABLE 2

Crosses to determine mating allele of meiotic segregants

\begin{tabular}{|c|c|c|c|}
\hline $\begin{array}{c}\text { Cross yielding } \\
\text { meiotic segregants } \\
A^{b} \times \text { Pan } 2-4\left(A^{7}\right)\end{array}$ & $\begin{array}{c}\text { Result of } \\
\text { test crosses } \\
\text { to } \begin{array}{c}\text { Pan } 2-4\left(A^{7}\right) \\
n=20\end{array}\end{array}$ & $\begin{array}{l}\text { Result of } \\
\text { test crosses } \\
\text { to } A^{2} \\
n=20\end{array}$ & $\begin{array}{l}\text { Mating allele } \\
\text { of segregant }\end{array}$ \\
\hline 1 & 20 & 0 & $A^{2}$ \\
\hline 2 & $2^{a}$ & 20 & Pan 2-4 $\left(A^{7}\right)$ \\
\hline 3 & $3^{a}$ & 20 & Pan 2-4 $\left(A^{7}\right)$ \\
\hline 4 & $18^{b}$ & 0 & $A^{2}$ \\
\hline 5 & 20 & 0 & $A^{2}$ \\
\hline 6 & 20 & 0 & $A^{2}$ \\
\hline 7 & 20 & 0 & $A^{2}$ \\
\hline 8 & $2^{a}$ & 20 & $\operatorname{Pan} 2-4\left(A^{7}\right)$ \\
\hline 9 & 20 & 0 & $A^{2}$ \\
\hline 10 & 0 & 20 & Pan 2-4 $\left(A^{7}\right)$ \\
\hline 11 & 20 & 0 & $A^{2}$ \\
\hline 12 & 0 & 20 & Pan 2-4 $\left(A^{7}\right)$ \\
\hline
\end{tabular}

${ }^{a}$ Expected frequency of selfing in common mating-type cross.

${ }^{b}$ Loss of compatibility sometimes observed in myxomycete clones.

TABLE 3

Crosses to determine mating allele of meiotic segregants

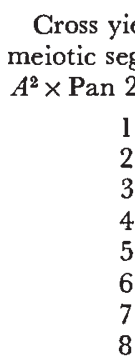

$$
\begin{array}{cc}
\text { Result of } & \text { Result of } \\
\text { test crosses } & \text { test crosses } \\
\text { to Pan } 2-7\left(A^{8}\right) & \text { to } A^{2} \\
n=20 & n=20
\end{array}
$$$$
\begin{gathered}
20 \\
20 \\
20 \\
2^{a} \\
2^{a} \\
0 \\
0 \\
0
\end{gathered}
$$

0

0

0

20

20

20

$17^{b}$

20
Mating allele of segregant

$A^{2}$
$A^{2}$
$A^{2}$
$2-7\left(A^{8}\right)$
$2-7\left(A^{8}\right)$
$2-7\left(A^{8}\right)$
$2-7\left(A^{8}\right)$
$2-7\left(A^{8}\right)$

$A^{2}$$$
A^{2}
$$

Pan 2-7 $\left(A^{8}\right)$

Pan $2-7\left(A^{8}\right)$

Pan $2-7\left(A^{8}\right)$

Pan 2-7 $\left(A^{8}\right)$

a Expected frequency of selfing in common mating-type cross.

${ }^{b}$ Loss of compatibility sometimes observed in myxomycete clones.

spores, clones of myxamoebae which arose from the spores were crossed with myxamoebae bearing the original mating type alleles, i.e. clones from the Pan 2-4 $\left(A^{7}\right) \times A^{2}$ cross were mated with Pan 2-4 $\left(A^{7}\right)$ and $A^{2}$ clones respectively, etc. The results of these analyses are shown in tables 2 and 3 . The differences in numbers of clones is due to the differential viability of spores resulting from the crosses. The percentages of viability for the Pan 2-4 $\left(A^{7}\right) \times A^{2}$ and Pan 2-7 $\left(A^{8}\right) \times A^{2}$ crosses were respectively 42 and 28 per cent. Plasmodia which appeared in common mating type crosses (apparently as the result of selfing) were allowed to sporulate, and the spores then analysed for mating type. This was necessary to insure that no new or mutant mating 
types had appeared spontaneously. Such analyses demonstrated that only the anticipated mating type was present, i.e. spore isolate number 2 from the Pan 2-4 $\left(A^{7}\right) \times A^{2}$ cross (table 3 ) possessed only the original mating allele borne by the Pan 2-4 $\left(A^{7}\right)$ isolate. In all cases only the initial two mating types used in each cross were recovered from the spore isolates.

Replica platings of these meiotic segregants were then carried out. Fifty such platings were made from each clone of myxamoebae. The frequency of selfing in the meiotic segregants is recorded in table 4 . It can be seen that clones bearing the $A^{2}$ mating allele now have the capacity to produce plasmodia without the necessity of being crossed with clones of compatible mating types. Also, it is seen that in both cases the frequency of selfing in the other mating types, Pan 2-4 $\left(A^{7}\right)$ and Pan 2-7 $\left(A^{8}\right)$, is markedly decreased in the meiotic segregants.

\section{TABLE 4}

Frequency of selfing in meiotic segregants resulting from cross with $A^{2}$

$\begin{array}{cccc}\text { Cross } & n & \text { Frequency of selfing } & \mathrm{P}^{a} \\ \text { Pan 2-4 }\left(A^{7}\right) \times A^{2} & 50 & \text { Pan 2-4 }\left(A^{7}\right)=1.2 \% ; A^{2}=7.1 \% & <0.01 \\ \text { Pan 2-4 }\left(A^{8}\right) \times A^{2} & 50 & \text { Pan 2-7 }\left(A^{8}\right)=0.8 \% ; A^{2}=3.3 \% & <0.01\end{array}$

a Chi-square analysis. Expected value based on equal distribution of selfing factor from $P$ to $F_{1}$ meiotic segregants.

\section{Discuussion}

Clonal development of plasmodia, "selfing", in the heterothallic myxomycete $D$. iridis was first reported by Collins, in 1961. In that paper and in a subsequent report (Collins, 1965), two hypotheses were presented for this observed variation from the normal compatibility mechanism. First, a small number of binucleate spores are formed which bear both mating alleles carried by the heterozygotic plasmodial nuclei. Binucleate, albeit haploid, spores of this type would of course produce clones of myxamoebae that mate legitimately. Second, a small fraction of a clone of myxamoebae undergo a spontaneous mutation at the mating locus, resulting in a mixed mating type population of myxamoebae. Again, legitimate mating is the result. Careful genetic analyses (Collins, 1961, 1965) have indicated that both hypotheses are indeed correct. In 1968, however, Collins and Ling reported that an analysis of compatibility in the Honduran clones bearing the mating alleles $A^{1}, A^{2}, A^{3}$ and $A^{4}$ gave results that were in disagreement with either of the two preceding hypotheses. It seemed clear from the 1968 study that another mechanism, which Collins termed " selfing" is considerably more important for bypassing the normal mechanism of cell compatibility. In the case of selfing, plasmodia occasionally arise in clones bearing only one mating allele, or in common mating type crosses, and the spores which result from the sporulation of these selfed plasmodia bear only the original mating type. Spontaneous mutations at the mating locus do not seem to occur, nor are spores formed which bear nuclei of more than one mating type. Clearly some mechanism other than the preceding two must be operational. Collins and Ling (1968) offered several hypotheses to explain the observed selfing phenomenon. One hypothesis, that of cytoplasmic inheritance, is easiest to reconcile with the data presented in this paper. 
Crosses between a non-selfing isolate, $A^{2}$, and isolates which bear different mating alleles and self in variable frequencies, Pan 2-4 $\left(A^{7}\right)$ and Pan 2-7 $\left(A^{8}\right)$, result in spores which inherit the selfing factor in a manner that appears to be independent of the inheritance of the mating allele. Clearly this is best explained by a reassortment of some cytoplasmic factor, not unlike the inheritance of cytoplasmic factors found in yeast (Wright and Lederberg, 1957) and filamentous fungi (Arlett, Grindle and Jinks, 1962). The variable inheritance observed, table 4 , in which the meiotic segregants displayed different frequencies of selfing may be due to chromosomal genes in the Panamanian isolates which suppress the phenotypic expression of the cytoplasmically transmitted factor. Nuclear control of the phenotypic expression of cytoplasmic factors has been previously reported in the yeast supersuppression system (Young and Cox, 1971). In the case of mating in D. iridis one could envisage a system in which extrachromosomal factors are distributed more or less randomly during spore cleavage, however, some mating types bear an allele or alleles very closely linked to the mating locus that have the function of modifying the usual phenotypic expression of that factor. The frequency of selfing among the individual spore isolates is shown in fig. 1 .
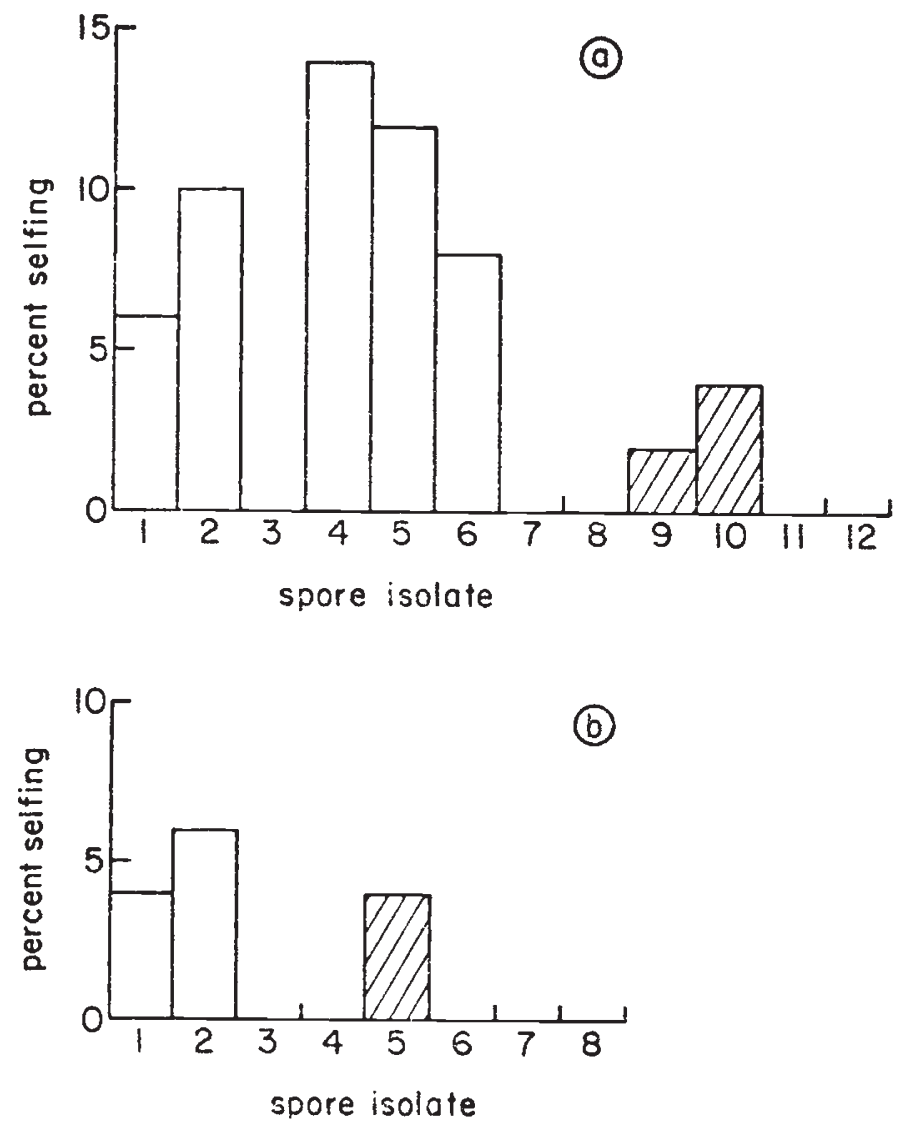

Fig. 1.- (a) Frequency of selfing in spores isolated from Pan 2-4 $\left(A^{7}\right) \times A^{2}$ cross: spores 1-7, $A^{2}$; spores 8-12, Pan 2-4 $\left(A^{7}\right)$. (b) Frequency of selfing in spores isolated from $\mathrm{Pan} 2-7$ $\left(A^{8}\right) \times A^{2}$ cross: spores $1-3, A^{2}$; spores $4-8$, Pan $2-7\left(A^{8}\right)$. 
Spores isolated from the cross $A^{2} \times \operatorname{Pan} 2-4\left(A^{7}\right)$ are represented by Fig. la. It is seen that clones arising from some spores, i.e. isolates 3 and 7 bearing the mating allele $A^{2}$ and isolates 7,11 and 12 bearing the mating allele $A^{7}$, do not self in the initial replica platings. Also, the frequency of selfing among different spore isolates is variable. For instance, replica platings of clones from spore isolates 1 through 7 , which bear the mating allele $A^{2}$, gave selfing frequencies from 0 to 14 per cent, whereas the frequency of selfing in the $A^{7}$ isolates varied from 0 to 4 per cent. These observations appear to substantiate the hypothesis that the selfing factor is inherited as a non-Mendelian gene. Were the factor inherited as a chromosomal gene one might expect that cells arising from single spores, which are haploid, would either all self, i.e. be essentially homothallic, or all be self-sterile; that is, all cells of a clonal population would be of the same genotype. Even if back mutations were to occur, they would presumably be of such low frequency that they could not possibly account for the variable frequencies of selfing observed among the individual isolates. If, for instance, spore isolate number 1 , which bears the mating allele $A^{2}$, were to have inherited a chromosomal gene that results in selfing, then one would expect that all fifty replica platings from clone number 1 would self. Our observation was that selfing occurred in only 6 per cent of the ititial platings. Only an unequal distribution of some cytoplasmic factor could account for these observations. The variable frequency of selfing found among the clones may result from a combination of unequal distribution of the factor in the spores during cleavage, the relatively short doubling time of the myxamoebae, approximately 5.5 hours, and unequal distribution of the factor during clonal mitoses. Replication of nuclear DNA, and subsequent cell division presumably occurs at a rate that prevents an equal distribution of the factor during clonal development.

The failure of the Honduran isolate $\left(A^{2}\right)$ to self until crossed with a Panamanian selfing isolate may simply be due to the fact that the selfing factor had not been previously transferred to the apparently more sensitive $A^{2}$ isolate. Once transferred the factor should then be expressed in a greater frequency in the $A^{2}$ mating type, which does not have the allele that confers resistance to the expression of the cytoplasmic factor. The data presented in table 4 supports this hypothesis. Viral induced plaques in the basidiomycete Schizophyllum commune appear to follow much the same pattern of inheritance (Koltin et al., 1973). To test this hypothesis one should reclone $\mathrm{F}_{1}$ spores produced from crosses between the Honduran isolate $A^{2}$ and the Panamanian isolates Pan 2-4 $\left(A^{7}\right)$ and Pan 2-7 $\left(A^{8}\right)$. The expectation would be that the frequency of selfing should now increase in the more sensitive $A^{2}$ isolate. Spores bearing the mating allele $A^{2}$, which were meiotic segregants from the crosses $A^{2} \times \operatorname{Pan} 2-4\left(A^{7}\right)$ and $A^{2} \times \operatorname{Pan} 2-7\left(A^{8}\right)$, do produce homozygotic plasmodia when recloned. These plasmodia then sporulate in the manner typical of selfed plasmodia. The frequency of selfing is observed to increase in subsequent generations, with average values of 76 and 100 per cent recorded for the $F_{2}$ and $F_{3}$ generations respectively. Spores cleaved in the $\mathrm{F}_{3}$ sporangia were then isolated and tested for mating type by crossing them with the original parental clones. Results from these crosses demonstrated that such spores bore only the original $A^{2}$ mating allele. Effectively the Honduran $A^{2}$ isolate now behaves in a manner very much similar to the homothallic Philippine-l isolate of $D$. iridis. This observed increase in the frequency of selfing in the Honduran isolate bearing the 
mating allele $A^{2}$ appears to substantiate the hypothesis for a variable sensitivity to a cytoplasmic factor which promotes plasmodial formation.

Finally, it should remain clear that we do not propose this to be the normal mechanism for plasmodial formation in the myxomycetes. The actual function of the mating allele has not as yet been defined. Perhaps the mating locus functions in a manner which is essentially that of an antigen-antibody system. This mechanism has been proposed by Prévost (1962) for compatibility in the basidiomycete Coprinus radiatus. Implicit in this compatibility system is the importance of the cell membrane. The important event antecedent to plasmodial formation would be cell fusion, regardless of whether it resulted in the formation of a heterokaryon, and eventually a heterozygote, or a homokaryon, and thus a homozygote. One might view the cytoplasmically inherited factor as being a virus which promotes cell fusion. Viral promoted cell fusions are of course well documented in the vertebrate cell culture systems (Harris et al., 1966), but as yet unrecorded in the myxomycetes. Also, viral genes are known to produce specific membrane alterations in transformed cells (Eckhart et al., 1971). Both factors, membrane alteration and cell fusion, could be of considerable importance in the selfing mechanism in the myxomycetes.

\section{References}

ARLETT, C. F., GRINDLE, M., AND JINKS, J. L. I962. The " red" cytoplasmic variant of Aspergillus nidulans. Heredity, 17, 197-209.

Collins, o. R. 1961. Heterothallism and homothallism in two Myxomycetes. Amer. F. Bot., $48,674-683$.

Collins, o. R. 1962. Mating types in the slime mold Physarum pusillum. Amer. F. Bot., 49, 659.

Collins, O. R. 1963. Multiple alleles at the incompatibility locus in the myxomycete Didymium iridis. Amer. F. Bot., 50, 477-480.

Collins, O. R. 1965. Evidence for a mutation at the incompatibility locus in the slime mold, Didmium iridis. Mycologia, 57, 314-315.

COLLINS, O. R., AND LING, H. 1964." Further studies in multiple allelomorph heterothallism in the myxomycete Didymium iridis. Amer. F. Bot., 51, 315-317.

COLLINS, O. R., AND LING, H. 1968. Clonally-produced plasmodia in heterothallic isolates of Didymium iridis. Mycologia, 60, 858-868.

DEE, J. 1962. Recombination in a myxomycete, Physarum polycephalum Schw. Genet. Res., Camb., 3, 11-23.

$\mathrm{DEE}$, J. 1966. Multiple alleles and other factors affecting plasmodial formation in the true slime mold Physarum polycephalum Schw. J. Protozool., 13, 610-616.

eckhart, w., DUlbecco, R., AND BURGer, M. M. 1971. Temperature-dependent surface changes in cells infected or transformed by a thermosensitive mutant of Polyoma virus. Proc. Nat. Acad. Sci., U.S.A., 68, 283-286.

GEHENIO, P. M., AND LUYET, B. J. 1950. Complete development of a mycetozoan from a single spore or a single myxamoeba. Biodyn., 7, 11-23.

HARRIS, H., WATKINS, J. F., FORD, C. E., AND SCHOEFL, G. I. 1966. Artificial heterokaryons of animal cells from different species. 7. Cell Sci., 1, 1-30.

HENNEY, M. R. 1967. The mating type system in the myxomycete Physarum flavicomum. Mycologia, 59, 637-652.

HENNEY, M. R., AND HENNEY, H. 1968. The mating-type systems of the myxomycetes Physarum rigidum and Physarum flavicomum. 7. Gen. Microbiol., 53, 321-332.

KERR, N. s. 1961. A study of plasmodium formation by the true slime mold Didymium nigripes. Exp. Cell Res., 23, 603-6I I.

KERR, N. s. 1967. Plasmodial formation by a minute mutant of the true slime mold, Didymium nigripes. Exp. Cell Res., 45, 646-655.

KOLTIN, Y., BERICK, R., STAMBERG, J., AND BEN-Shaul, y. 1973. Virus-like particles and cytoplasmic inheritance in a higher fungus. Nature New Biol., 241, 108-109. 
MUKHERJEE, K. L., AND ZABKA, G. G. 1964. Studies of multiple allelism in the myxomycete Didymium iridis. Can. J. Bot., 42, 1459-1466.

prévost, G. 1962. Étude génétique d'un basidiomycete: Coprinus radiatus. Thesis, Univ. de Paris, Paris.

WRIGHT, R. E., AND LEDERBERG, J. 1957. Extranuclear transmission in yeast heterokaryons. Proc. Nat. Acad. Sci., U.S.A., 43, 919-923.

YEMMA, J. J., AND THERRIEN, C. D. 1972. Quantitative microspectrophotometry of nuclear DNA in selfing strains of the myxomycete Didymium iridis. Amer. 7. Bot., 59, 828-835. YOUNG, c. s. H., AND Cox, B. s. 1971. Extrachromosomal elements in a super-suppression system of yeast. 1. A nuclear gene controlling the inheritance of the extrachromosomal elements. Heredity, 26, 413-422. 